\title{
Р.А. НАКОНЕЧНЫЙ
}

\section{ПРОГНОСТИЧЕСКИЕ МАРКЕРЫ ЭНДОВЕЗИКАЛЬНОГО ЛЕЧЕНИЯ ПУЗЫРНО-МОЧЕТОЧНИКОВОГО РЕФЛЮКСА У ДЕТЕЙ}

\author{
Львовский национальный медицинский университет имени Данила Галицкого, \\ Украина
}

Цель. Определить цистоскопические прогностические маркеры эффективности лечения детей с различными клиническими формами пузырно-мочеточникового рефлюкса (ПМР) для адекватного ведения пациентов и коррекции порока.

Материал и методы. У 270 пациентов с различными формами ПМР II-IV степеней в возрасте от 6 месяцев до 14 лет во время эндовезикального лечения проанализирована структура устьев мочеточников. Через полгода после эндовезикального лечения цистоскопическая картина оценена у 64 пациентов с ПМР I-IV степеней, у которых степень ПМР сохранилась или снизилась. Изучали положение «вулканообразного» выпячивания, миграцию и экструзию имплантата, а при наличии выпячивания имплантата - степень гидродилятации распластанного на нем устья мочеточника.

Результаты. Через полгода после однократного введения имплантата эффективность миниинвазивного лечения ПМР II степени составила $87,84 \%$, III - 83,45\% и IV - 39,22\%. А результативность повторного эндовезикального лечения ПМР I-IV степеней, с учетом снижения степени порока после первого вмешательства, достигла 57,81\%, что в целом дало прирост по выздоровлению на 13,7\%. В общем эффективность миниинвазивного вмешательства поднялась до 90\%. Отсутствие «вулканообразного» выпячивания после первого эндовезикального вмешательства оказалось выраженным отрицательным прогностическим маркером относительно эффективности повторного введения имплантата $-\mathrm{OR}=0,24[0,07-0,9], \mathrm{RR}=0,36$ [0,12-1,05], а миграция имплантата в латеральном направлении менее выраженным отрицательным маркером $-\mathrm{OR}=0,76$ [0,31-1,85], $\mathrm{RR}=0,84$ [0,46-1,51]. Уменьшение «вулканообразного» выпячивания в объеме и смещение его в медиальном направлении оказались положительными прогностическими маркерами относительно эффективного повторного введения имплантата. Ассоциация была более значительной при потере объема имплантата: $\mathrm{OR}=2,08[0,82-5,28], \mathrm{RR}=1,54[0,92-2,57]$ по сравнению с $\mathrm{OR}=1,71$ [0,69-4,25] и $\mathrm{RR}=1,39$ [0,82-2,34] при миграции валика к срединной линии мочевого пузыря.

Заключение. При планировании повторного эндовезикального лечения ПМР необходим персонифицированный подход к пациенту с учетом прогностических цистоскопических маркеров.

Ключевые слова: пузырно-мочеточниковый рефлюкс, эндоскопия, цистоскопия, мочевой пузырь, прогностический цистоскопический маркер, лечение, конечный результат

Objectives. To determine cystoscopic prognostic markers of treatment effectiveness in children with different clinical forms of vesicoureteral reflux (VUR) for an adequate patients' treatment and defect correction.

Methods. The patients $(n=270)$ with various forms of VUR of II-IV grades at the age from 6 months up to 14 years the structure of the ureter ostium was analyzed during the endovesical treatment. Half a year after the endovesical treatment a cystoscopic picture was evaluated in 64 patients with VUR of I-IV grades in whom VUR of stage remained in the same condition or decreased. The position of the "volcano shape" protrusion, migration and extrusion of the implant and if the implant bulged - the hydrodilatation grade of the ureteral orifice sprawled on it were studied.

Results. Six months after a single implantation the effectiveness of the minimally invasive treatment of VUR (II grades) was $87,84 \%$, of III $-83,45 \%$ and of IV $-39,22 \%$. The effectiveness of endovesical VUR re-treatment of I-IV grades reached $57,81 \%$, which in general made gain on recovery by $13,7 \%$. Generally, minimally invasive intervention efficiency has risen up to $90 \%$. The lack of "volcano shape" protrusion after the first endovesical procedure turned out to be an evident negative prognostic marker regarding the effectiveness of repeated implantation $\mathrm{OR}=0,24[0,07-0,9], \mathrm{RR}=0,36[0,12-1,05]$, and the migration of the implant in the lateral direction was less pronounced negative marker $-\mathrm{OR}=0,76[0,31-1,85], \mathrm{RR}=0,84[0,46-1,51]$.

Reducing the "volcano shape" protrusion and its displacement to the medial direction are positive prognostic markers regarding the effective re-implantation. The association was more significant with implant volume loss: $\mathrm{OR}=2,08[0,82-5,28], \mathrm{RR}=1,54[0,92-2,57]$ if compared with $\mathrm{OR}=1,71[0,69-4,25]$ and $\mathrm{RR}=1,39[0,82-2,34]$ in case of migration to the midline of the bladder.

Conclusion. While planning the endovesical re-treatment of VUR the personalized approach to a patient is necessary considering the prognostic cystoscopic markers.

Keywords: vesicoureteral reflux, endoscopy, cystoscopy, bladder, prognostic cystoscopic markers, treatment, final results

Novosti Khirurgii. 2017 Jan-Feb; Vol 25 (1): 53-59

Prognostic Markers of Endovesical Treatment of Vesicoureteral Reflux in Children

R.A. Nakonechnyy 


\section{Введение}

Пузырно-мочеточниковый рефлюкс (ПМР) занимает ведущее место в структуре детской урологической патологии $[1,2,3]$. Его частота варьирует от 1 до $3 \%$ в популяции детского населения $[4,5]$.

Обычно рецидивирующее течение инфекции мочевых путей при ПМР и даже смазанная клиника с длительным бессимптомным течением порока приводят к развитию тяжелых осложнений. Среди них следует выделить рефлюкснефропатию, наибольший риск формирования которой наблюдается у детей двухлетнего возраста. Рефлюкс-нефропатия имеет тенденцию к прогрессированию и нефросклерозу, даже несмотря на длительную клинико-лабораторную ремиссию пиелонефрита, низкую степень ПМР или успешно выполненную операцию. Прогрессирующий нефросклероз, артериальная гипертензия и хроническая почечная недостаточность (ХПН) приводят к инвалидности пациентов в молодом возрасте $[6,7,8]$.

Изложенные факты обусловливают необходимость ранней диагностики ПМР, своевременного и адекватного лечения заболевания. Однако продолжаются дискуссии о подходах к лечению ПМР, поиску новых и усовершенствованию существующих методов его коррекции с уточнением показаний к вмешательству. Так, дискуссионной остается эффективность длительной антибактериальной терапии порока. А к методикам хирургической коррекции ПМР выставляются требования на максимально быстрое выздоровление при минимальной инвазивности и травматичности с предупреждением осложнений и рецидивов. В этом контексте эндоскопическая коррекция ПМР с имплантацией обьемообразующего вещества на сегодняшний день наиболее соответствует этим требованиям. Она физиологична, поскольку сохраняет анатомическую целостность пузырно-мочеточникового сегмента и соответственно способствует его созреванию $[9,10,11,12]$. Нет также единого мнения о сроках и способах хирургического лечения ПМР разных степеней. Так, при высоких степенях ПМР различные способы уретероцистонеостомий превосходят по эффективности эндоскопическую коррекцию порока. В то же время при низких степенях рефлюкса получены хорошие результаты при эндоскопической инъекции имплантата под устье мочеточника [13]. К тому же родители больных детей отдают предпочтение эндоскопическому лечению в качестве «первой линии» при коррекции ПМР [3], даже несмотря на возможную необходимость реимплантации мочеточника после неэффективного эндоскопического лечения [14].
Именно неудовлетворительные результаты лечения ПМР побуждают к разработке четких показаний для эндовезикального метода коррекции порока и поиска высокочувствительных и специфических прогностических маркеров его эффективности [15].

Цель. Определить цистоскопические прогностические маркеры эффективности лечения детей с различными клиническими формами ПМР для адекватного ведения пациентов и коррекции порока.

\section{Материал и методы}

У 270 детей (388 мочеточников) с различными клиническими формами ПМР II-IV степеней в возрасте от 6 месяцев до 14 лет во время эндовезикального лечения проанализирована структура устьев мочеточников. Основную часть составили пациенты с ПМР ІІІ степени $145(53,7 \%)$ пациентов (202 мочеточника). Существенно меньше детей было с пороком II степени - 74 (27,41\%) (113 мочеточников). И еще менее многочисленной оказалась подгруппа ПМР IV степени - 51 (18,89\%) ребенок (73 мочеточника). Всем пациентам с ПМР II-IV степени уродинамику восстанавливали эндовезикальной коррекцией. Применяли такие миниинвазивные методики, как STING, HIT, Double HIT, а также при необходимости их комбинации. Способ вмешательства определяли интраоперационно в зависимости от степени ПМР и цистоскопической картины. В качестве имплантата использовали гидрофильные полиакриламидные гели. Во время проведения миниинвазивного вмешательства всем 270 пациентам с ПМР определяли расположение, форму, степень гидродилятации и сократительную способность устьев мочеточников.

Норму проанализировали у 22 детей без врожденных пороков мочевыделительной системы, преимущественно с подозрением на доброкачественные опухолевидные новообразования в проекции мочевого пузыря по данным УЗИ или компьютерной томографии - полипы и с невыясненной эритроцитурией, которая требовала уточнения.

Через полгода после эндовезикального лечения цистоскопическую картину оценили у 64 пациентов, у которых степень ПМР сохранилась или снизилась. Изучали положение «вулканообразного» выпячивания, миграцию и экструзию имплантата, а при наличии выпячивания имплантата - степень гидродилятации распластанного на нем устья мочеточника. В подгруппе, в которой степень ПМР осталась той же и соответственно «вулканообразное» 
выпячивание отсутствовало, оценивали конфигурацию устья, его положение и степень гидродилятации.

При статистических расчетах определяли относительный риск (RR), отношение шансов (OR) и доверительные интервалы (ДИ) с вероятностью 95\% [95\% ДИ]. Показатель подавали в формате RR [L-U], где L - нижняя и $\mathrm{U}$ - верхняя граница ДИ. Для RR определяли чувствительность (Se) и специфичность (Sp). Оценку статистической достоверности различий показателей в группах проводили по одно- и двусторонним тестам точного критерия Фишера при вероятности безошибочного прогноза не менее $95 \%$.

\section{Результаты}

До операции. Анализ полученных результатов показал, что у пациентов с ПМР к нехарактерным цистоскопическим признакам можно отнести расположение устьев мочеточников в зоне А с гидродилятацией $\mathrm{H}_{0}$ степени и активной перистальтикой. По этим критериям выявлены негативная ассоциация с ПМР и статистически значимые различия по двустороннему точному критерию Фишера относительно показателей у практически здоровых детей $-\mathrm{p}<0,001$.

По цистоскопической картине у пациентов с ПМР можно утверждать, что для них характерны устья мочеточников со следующими параметрами: форма - «стадион», «подкова» и «ямка для гольфа»; локализация - смещение в зону В и более латерально на боковую стенку мочевого пузыря; степень гидродилятации - Н1 и выше; перистальтика вялая. Эти показатели характеризовались положительной ассоциацией с ПМР и высокой специфичностью. Так, для стадионообразных устьев мочеточников специфичность достигала 0,91 , а для остальных вышеперечисленных цистоскопических признаков - 1,0. У подавляющего большинства детей с ПМР устья мочеточников имели вялую перистальтику - 93,33\%: RR=2,22 [1,58-3,13], $\mathrm{p}<0,001, \mathrm{Se}=0,93$ и $\mathrm{Sp}=1,0-$ со статистически значимыми различиями по двустороннему точному критерию Фишера относительно показателей у практически здоровых детей.

Через 6 месяцев после операции. Выздоровевшие. Через полгода после однократного введения имплантата эффективность миниинвазивного лечения ПМР II степени составила $87,84 \%$, III - 83,45\% и IV - 39,22\%. У пациентов с ПМР отмечена четкая тенденция к снижению результативности эндовезикального лечения при увеличении зияния устья мочеточника. В частности, эффективность вмешательства при устьях мочеточников в форме «стадиона» равнялась $97,17 \%$, «подковы» $-79,53 \%$ и «ямки для гольфа» $-5,41 \%$.

При устьях мочеточников в форме «стадиона» зарегистрирована положительная ассоциация с выздоровлением со статистически достоверными различиями к предоперационному периоду и низкими специфичностью и чувствительностью: $\mathrm{OR}=1,55$ [1,07-2,23], $\mathrm{RR}=1,28$ [1,04$1,57], \mathrm{Sp}=0,61, \mathrm{Se}=0,5, \mathrm{p}<0,03$, а в форме «ямки для гольфа», наоборот, выраженную негативную ассоциацию со статистически значимыми различиями к дооперационному периоду и средней специфичностью: $\mathrm{OR}=0,06$ [0,02-0,26], $\mathrm{RR}=0,11$ $[0,03-0,43], \mathrm{Sp}=0,86, \mathrm{p}<0,001$.

При смешении устьев мочеточников на боковую стенку мочевого пузыря снижалась эффективность эндовезикального лечения и особенно существенно начиная с зоны С. Так, при локализации устьев мочеточников в зоне А она составила $100 \%$, В - 94,81\%, C - 67,61\% и $\mathrm{D}-5,56 \%$. Размещение устьев мочеточников в зоне В характеризовалось положительной ассоциацией относительно выздоровления с достоверными различиями к показателям в дооперационном периоде по одностороннему точному критерию Фишера и средней чувствительностью: $\mathrm{OR}=1,83$ [1,25-2,69], $\mathrm{RR}=1,43$ $[1,13-1,81], \mathrm{p}<0,003, \mathrm{Se}=0,71$. Для локализации устьев мочеточников в зоне D была присуща выраженная негативная ассоциация со статистически значимыми различиями к дооперационному периоду и средней специфичностью $\mathrm{OR}=0,06[0,02-0,27], \mathrm{RR}=0,11[0,03-0,44]$, $\mathrm{p}<0,001, \mathrm{Sp}=0,87$.

У пациентов с гидродилятацией устьев мочеточников $\mathrm{H}_{0}$ степени наступило полное выздоровление, при $\mathrm{H}_{1}-95,95 \%, \mathrm{H}_{2}-53,33 \%$ и $\mathrm{H}_{3}-3,33 \%$. При гидродилятации устьев мочеточников $\mathrm{H}_{0}$ и $\mathrm{H}_{1}$ степеней зафиксирована положительная ассоциация с выздоровлением. Так, при гидродилятации устьев мочеточников $\mathrm{H}_{0}$ степени она была умеренной и характеризовалась высокой специфичностью: $\mathrm{OR}=1,32$ $[0,46-3,83], \mathrm{RR}=1,16[0,68-1,98], \mathrm{Sp}=0,97$, а при $\mathrm{H}_{1}$ - более выраженной со средней чувствительностью и статистически значимыми различиями к предоперационному периоду: $\mathrm{OR}=2,33$ [1,52$3,56], \mathrm{RR}=1,68$ [1,26-2,23], $\mathrm{Se}=0,81, \mathrm{p}<0,001$.

Зато у пациентов с гидродилятацией устьев мочеточников $\mathrm{H}_{2}$ и $\mathrm{H}_{3}$ степеней, наоборот, обнаружена негативная ассоциация с выздоровлением при средней специфичности тестов и достоверных различиях к дооперационному периоду. В частности, при $\mathrm{H}_{2}$ получены такие результаты: $\mathrm{OR}=0,64[0,24-0,4], \mathrm{RR}=0,81[0,61$ - 
$1,08], \mathrm{Sp}=0,78, \mathrm{p}<0,05$ и при $\mathrm{H}_{3}: \mathrm{OR}=0,04[0,01$ $0,29], \mathrm{RR}=0,07[0,01-0,48], \mathrm{Sp}=0,89, \mathrm{p}<0,05$.

Через 6 месяцев после операции - улучшение. Спустя полгода после эндовезикального лечения степень ПМР уменьшилась на один или несколько степеней более чем в десятой части пациентов (в 11,11\% случаев) и осталась на прежнем уровне в $12,59 \%$. В целом это $23,7 \%$ пациентов, у которых степень порока осталась без изменений или несколько снизилась.

У пациентов, у которых степень порока осталась на прежнем уровне или несколько снизилась, через 6 месяцев после миниинвазивного лечения ПМР не выявлено «вулканообразного» выпячивания более чем у четвертой части пациентов $-26,56 \%$. Среди них, в 70,59\% случаев, преобладали устья мочеточников в форме «ямки для гольфа» с размещением в зоне D в противовес $29,41 \%$ больных с устьями в форме «подковы» и расположением в области С.

В этой подгруппе пациентов с подковообразными устьями мочеточников $(\mathrm{OR}=0,1$ $[0,04-2,55], \mathrm{RR}=0,13[0,05-0,32], \mathrm{Sp}=0,53)$ и расположением в зоне $\mathrm{C}(\mathrm{OR}=0,26[0,1-0,67]$, $\mathrm{RR}=0,31[0,13-0,74], \mathrm{Sp}=0,75)$ получены крайне низкие показатели отношения шансов и относительного риска при сравнении с неоперированными пациентами, что указывает на возможность выздоровления.

Для устьев мочеточников в форме «ямки для гольфа» $(\mathrm{OR}=1,45$ [0,71-2,98], $\mathrm{RR}=1,34$ $[0,78-2,33], \mathrm{Sp}=0,86)$ и расположением в зоне $\mathrm{D}(\mathrm{OR}=2,35[1,1-5,02], \mathrm{RR}=1,38[0,8-2,38]$, $\mathrm{Sp}=0,86)$ была присуща умеренная положительная ассоциация со средней специфичностью, что свидетельствует о бесперспективности эндовезикального лечения у пациентов с этими параметрами устьев мочеточников и о вероятности сохранения ПМР.

Степень $\mathrm{H}_{1}$ гидродилятации устьев мочеточников характеризовалась негативной ассоциацией относительно сохранения ПМР после эндовезикального вмешательства: $\mathrm{OR}=0,64$ $[0,37-1,1], \mathrm{RR}=0,7[0,45-1,08]$ и $\mathrm{Se}=0,53$. Зато для более высоких степеней гидродилятации устьев мочеточников $-\mathrm{H}_{2}$ и $\mathrm{H}_{3}$ - была присуща положительная ассоциация. Так, у детей с ПМР при $\mathrm{H}_{2}$ : $\mathrm{OR}=1,59$ [0,87-2,9], $\mathrm{RR}=1,44$ [0,91-2,3], $\mathrm{Sp}=0,78$, а при $\mathrm{H}_{3}$ соответственно $1,48[0,68$ $3,21], 1,36[0,76-2,45]$ и 0,89 .

Уменьшение объема «вулканообразного» выпячивания зафиксировано у 18,75\% пациентов. Более чем у пятой части пациентов выявлено смещение имплантата к срединной линии мочевого пузыря $-21,88 \%$. И особенно часто имплантат мигрировал в латеральном направлении, где анатомически более рыхлый подслизистый слой стенки мочевого пузыря $32,81 \%$ пациентов.

Отсутствие «вулканообразного» выпячивания, уменьшение его объема, а также миграция имплантата в медиальном или латеральном направлениях характеризовались выраженной положительной ассоциацией на сохранение ПМР после эндовезикального лечения. При сравнении с дооперационным периодом специфичность перечисленных тестов самая высокая $-1,0$.

Повторная операция. Эффективность повторного эндовезикального лечения ПМР I-IV степеней составила $57,81 \%$, что в целом дало прирост по выздоровлению на 13,7\%. В общем эффективность миниинвазивного вмешательства повысилась до 90\%. После повторного эндовезикального вмешательства выздоровели больные ПМР исключительно I-III степеней. То есть у пациентов, у которых сохранился ПМР IV степени, повторное введение имплантата также не дало положительного результата. Эффективность повторного эндовезикального лечения снижалась с увеличением степени ПМР. В частности, при ПМР I степени она составила $100 \%$, II $-85,71 \%$ и III $-63,64 \%$.

Отсутствие «вулканообразного» выпячивания после первого эндовезикального вмешательства это выраженный отрицательный прогностический маркер относительно эффективности повторного введения имплантата. Это подтверждено низкими отношением шансов $(0,24[0,07-0,9])$ и относительным риском $(0,36[0,12-1,05])$ со статистически достоверными различиями к соответствующему показателю у пациентов после первого оперативного вмешательства $(\mathrm{p}<0,04)$ и средней специфичностью - 0,73.

Миграция имплантата в латеральном направлении - это менее выраженный отрицательный прогностический маркер относительно эффективности повторного эндовезикального вмешательства: $\mathrm{OR}=0,76$ [0,31-1,85], $\mathrm{RR}=0,84$ [0,46-1,51], $\mathrm{Sp}=0,67$.

А уменьшение «вулканообразного» выпячивания в объеме и смещение его в медиальном направлении выявились положительными прогностическими маркерами эффективного повторного введения имплантата. По отношению шансов и относительному риску положительная ассоциация более значительна при потере объема имплантата $-\mathrm{OR}=2,08$ [0,82-5,28], $\mathrm{RR}=1,54$ $[0,92-2,57], \mathrm{Sp}=0,81-$ по сравнению с $\mathrm{OR}=1,71$ $[0,69-4,25], \mathrm{RR}=1,39[0,82-2,34]$ и $\mathrm{Sp}=0,78$ при миграции валика к срединной линии мочевого пузыря.

Степень $\mathrm{H}_{1}$ гидродилятации устьев мочеточников, которую визуализировали у 91,18\% 
пациентов после первого эндовезикального вмешательства, оказалась положительным прогностическим маркером эффективности повторного введения имплантата. Получена выраженная положительная ассоциация по $\mathrm{OR}=4,56$ [1,67-12,43] и $\mathrm{RR}=2,86$ [1,32-6,2] со средней $\mathrm{Sp}=0,81$ и статистически значимыми различиями относительно пациентов, у которых ПМР сохранился или снизился после первого эндовезикального вмешательства $\mathrm{p}<0,003$. Зато степень $\mathrm{H}_{2}$ гидродилятации устьев мочеточников явилась отрицательным прогностическим маркером эффективности повторного эндовезикального лечения ПМР. Она характеризовалась заметной отрицательной ассоциацией по $\mathrm{OR}=0,43[0,15-1,18]$ и $\mathrm{RR}=0,56$ $[0,26-1,18]$ с низкой $\mathrm{Sp}=0,69$.

\section{Обсуждение}

У пациентов с ПМР устья мочеточников, которые имеют конфигурацию с более выраженными признаками зияния, размещаются значительно латеральнее от середины мочевого пузыря. Причем у пациентов с высшими степенями ПМР отмечена тенденция к форме устьев мочеточников с более выраженным зиянием, а соответственно и степенью гидродилятации и латерализации на боковую стенку мочевого пузыря.

После однократной эндовезикальной коррекции ПМР у детей отсутствие «вулканообразного» выпячивания может быть результатом банальной экструзии имплантата или его миграции за пределы визуализации, а именно по ходу мочеточника вследствие существенной экстравезикализации последнего. То есть, у этих пациентов устья рефлюксирующих мочеточников имеют конфигурацию с более выраженными признаками зияния и соответственно латерализации. В таких случаях образуется кольцо имплантата в подслизистой оболочке мочеточника, ширина которого соизмерима с объемом введенного препарата. Понятно, что такое расположение имплантата не создает антирефлюксной защиты. Итак, если у пациентов с ПМР устья рефлюксирующих мочеточников имеют конфигурацию с выраженными признаками зияния и соответственно латерализации, то отсутствие «вулканообразного» выпячивания через полгода после эндовезикальной коррекции порока следует считать неблагоприятным прогностическим маркером относительно повторных миниинвазивных вмешательств. Исключение могут составлять только случаи технических ошибок при первом эндовезикальном вмешательстве или убедительных признаках экструзии им- плантата. К таким можно отнести наблюдения родителей: «желеобразные» включения в моче после эндовезикальной коррекции ПМР.

Феномен уменьшения объема «вулканообразного» выпячивания является следствием частичной экструзии или «самоорганизации» имплантата. Уменьшение в объеме «вулканообразного» выпячивания можно оценить исключительно при адекватном расположении имплантата под устьем мочеточника и когда увеличивается щель входа в мочеточник. При миграции имплантата медиально или латерально визуально верно определить объем имплантата нереально. Миграция имплантата происходит вследствие значительной рыхлости подслизистого слоя стенки мочевого пузыря, который образован соединительной тканью с большим количеством кровеносных и лимфатических сосудов. Особенно это касается случаев, когда рефлюксирующее устье мочеточника смещено на боковую стенку мочевого пузыря, а именно в зоны C и D. Подтверждением этому может служить более частая миграция имплантата в латеральном направлении. Зато в зоне A, а именно при типичном расположении устьев мочеточников на верхушке мочепузырного треугольника, слизистая, наоборот, фиксирована к мышечному шару. Поэтому имплантату более сложно мигрировать в медиальном направлении, и не всегда для этого есть место в подслизистом слое.

Уменьшение ПМР на один или несколько степеней через полгода после эндовезикальной коррекции при улучшении клинического течения в послеоперационном периоде, а именно отсутствие или уменьшение частоты обострений инфекции мочевыводящих путей; отсутствие повышения температуры тела, болевых и дизурических симптомов (адекватный ритм мочеиспусканий); нормализация лабораторных показателей - анализов мочи и маркеров склерозирования паренхимы почки; улучшение уродинамики и функции почки с восстановлением темпов ее роста, надо оценивать как положительный результат миниинвазивного вмешательства.

\section{Заключение}

У пациентов с ПМР II-IV степени целесообразно использовать эндовезикальную коррекцию. Это альтернатива выбора в подходах к быстрому и эффективному устранению порока.

Эндоскопическое лечение ПМР при необходимости, можно проводить повторно без серьезных проблем, которые присущи традиционным хирургическим вмешательствам. 
При планировании повторного эндовезикального лечения ПМР необходим персонифицированный подход к пациенту с учетом положительных прогностических цистоскопических маркеров относительно выздоровления, среди которых следует выделить уменьшение в объеме «вулканообразного» выпячивания или его смещение в медиальном направлении и $\mathrm{H} 1$ степень гидродилятации устьев мочеточников.

\section{Работа выполнялась в соответствии с планом научных исследований Львовского националь- ного медицинского университета имени Данила Галицкого.}

\section{ЛИТЕРАТУРА}

1. Лесовой ВН, Туренко ИА, Дубинина АН. Оценка отдаленных результатов эндоскопической коррекции пузырно-мочеточникового рефлюкса Eкcneриментальна і Клінічна Медицина. 2009;(3):128-31.

2. Сеймівський ДА. Вроджені вади сечоводу у дітей. Природная Медицина. 2011 Сент;(7):14-15.

3. Straub J, Apfelbeck M, Karl A, Khoder W, Lellig $\mathrm{K}$, Tritschler $\mathrm{S}$, et al. Vesico-ureteral reflux: diagnosis and treatment recommendations. Urologe A. 2016 Jan;55(1):27-34. doi: 10.1007/s00120-015-0003-3. [Article in German]

4. Crozier J, Aw I, Tan PH, Clarke D. Taking the STING out of ureteral obstruction. J Endourol Case Rep. 2016 Oct 1;2(1):166-68. eCollection 2016.

5. Khawaja MA, Nawaz G, Jamil MI, Muhammad S, ur Rehman A, Shohab D, et al. Efficacy of endoscopic treatment for primary vesicoureteric refluxin children. $J$ Ayub Med Coll Abbottabad. 2015 Oct-Dec;27(4):861-64. 6 . Lee LC, Lorenzo AJ, Koyle MA. The role of voiding cystourethrography in the investigation of children with urinary tract infections. Can Urol Assoc J. 2016 May-Jun;10(5-6):210-14.

7. Arlen AM, Cooper CS. Controversies in the management of vesicoureteral reflux. Curr Urol Rep. 2015 Sep;16(9):64. doi: 10.1007/s11934-015-0538-2. Review.

8. Puri P, Hunziker M. Chapter 66: Vesicoureteral reflux. In: Ziegler MM, Azizkhan RG, von Allmen D, Weber TR, editors. Operative Pediatric Surgery. 2nd ed. McGraw-Hill Education; 2014. p. 818-27.

9. Kirsch AJ, Perez-Brayfield M, Smith EA, Scherz HC. The modified sting procedure to correct vesicoureteral reflux: improved results with submucosal implantation within the intramural ureter. J Urol. 2004 Jun;171(6 Pt 1):2413-16.

10. Chertin B, Puri P. Endoscopic management of vesicoureteral reflux: does it stand the test of time? Eur Urol. 2002 Dec;42(6):598-606; discussion 606.

11. Elder JS, Shah MB, Batiste LR. Part 3: Endoscopic injection versus antibiotic prophylaxis in the reduction of urinary tract infections in patients with vesicoureteral reflux. Curr Med Res Opin. 2007;23(Suppl 4):S15-20.

12. Dogan HS, Altan M, Citamak B, Bozaci AC, Koni A, Tekgul S. Factors affecting the success of endoscopic treatment of vesicoureteral reflux and comparison of two dextranomer based bulking agents: does bulking substance matter? J Pediatr Urol. 2015 Apr;11(2):90.e15. doi: 10.1016/j.jpurol.2014.12.009.
13. Tekgül S, Dogan HS, Erdem E, Hoebeke P, Kocvara R, Nijman JM, Radmayr C, et al. Guidelines on paediatric urology [Electronic Resource]. 2015. 130 p. Available from: http://uroweb.org/wp-content/uploads/EAU-Guidelines-Paediatric-Urology-2015.pdf.

14. Lee S, Jeong SC, Chung JM, Lee SD. Secondary surgery for vesicoureteral reflux after failed endoscopic injection: Comparison to primary surgery. Investig Clin Urol. 2016 Jan;57(1):58-62. doi: 10.4111/ icu.2016.57.1.58.

15. Lee EK, Gatti JM, Demarco RT. Long-term followup of dextranomer/hyaluronic acid injection for vesicoureteral reflux: late failure warrants continued followup. J Urol. 2009;181:1869-74. discussion 1874-75. doi: 10.1016/j.juro.2008.12.005.

\section{REFERENCES}

1. Lesovoi VN, Turenko IA, Dubinina AN. Otsenka otdalennykh rezul'tatov endoskopicheskoi korrektsii puzyrno-mochetochnikovogo refliuksa [Evaluation of longterm results of endoscopic correction of vesicoureteral reflux]. Eksper i Klin Meditsina. 2009;3:128-31.

2. Seimivs'kii DA. Vrodzheni vadi sechovodu u ditei [Congenital ureter in children]. Prirod Meditsina. 2011 Sent;(7):14-15.

3. Straub J, Apfelbeck M, Karl A, Khoder W, Lellig $\mathrm{K}$, Tritschler $\mathrm{S}$ et al. Vesico-ureteral reflux: diagnosis and treatment recommendations. Urologe A. 2016 Jan;55(1):27-34. doi: 10.1007/s00120-015-0003-3. Review. German.

4. Crozier J, Aw I, Tan PH, Clarke D. Taking the STING out of ureteral obstruction. J Endourol Case Rep. 2016 Oct 1; 2(1):166-68.

5. Khawaja MA, Nawaz G, Jamil MI, Muhammad S, ur Rehman A, Shohab D, et al. Efficacy of endoscopic treatment for primary vesicoureteric reflux in children. J Ayub Med Coll Abbottabad. 2015 OctDec;27(4):861-64.

6. Lee LC, Lorenzo AJ, Koyle MA. The role of voiding cystourethrography in the investigation of children with urinary tract infections. Can Urol Assoc J. 2016 MayJun;10(5-6):210-14.

7. Arlen AM, Cooper CS. Controversies in the management of vesicoureteral reflux. Curr Urol Rep. 2015 Sep;16(9):64. doi: 10.1007/s11934-015-0538-2. Review. 8. Puri P, Hunziker M. Vesicoureteral reflux. In: Ziegler MM, Azizkhan RG, von Allmen D, Weber TR, editors. Operative Pediatric Surgery. 2nd ed. McGraw-Hill Education; 2014. p. 818-27.

9. Kirsch AJ, Perez-Brayfield M, Smith EA, Scherz HC. The modified sting procedure to correct vesicoureteral reflux: improved results with submucosal implantation within the intramural ureter. J Urol. 2004 Jun;171(6 Pt 1):2413-6.

10. Chertin B, Puri P. Endoscopic management of vesicoureteral reflux: does it stand the test of time? Eur Urol. 2002;42:598-606.

11. Elder JS1, Shah MB, Batiste LR, Eaddy M. Part 3: Endoscopic injection versus antibiotic prophylaxis in the reduction of urinary tract infections in patients with vesicoureteral reflux. Curr Med Res Opin. 2007 Sep;23(Suppl 4):S15-20.

12. Dogan HS, Altan M, Citamak B, Bozaci AC, Koni A, Tekgul S. Factors affecting the success of endoscopic treatment of vesicoureteral reflux and comparison of two dextranomer based bulking agents: does bulking substance matter? J Pediatr Urol. 2015 Apr; 11(2):90. 
e1-5. doi: 10.1016/j.jpurol.2014.12.009.

13. Tekgul S, Dogan HS, Erdem E, Hoebeke P, Kocvara

R, Nijman JM et al. Guidelines on paediatric urology.

Eur Assoc Urol. 2015; Available from: http://uroweb.

org/wp-content/uploads/EAU-Guidelines-Paediatric-

Urology-2015.pdf.

14. Lee S, Jeong SC, Chung JM, Lee SD. Secondary surgery for vesicoureteral reflux after failed endo-

\section{Адрес для корреспонденции}

79010, Украина, г. Львов,

ул. Пекарская, д. 69,

Львовский национальный медицинский университет имени Данила Галицкого, кафедра детской хирургии,

тел. раб.: + 38-032-293-97-39,

e-mail: nrostyslav@gmail.com,

Наконечный Ростислав Андреевич

\section{Сведения об авторах}

Наконечный Р.А., аспирант кафедры детской хирургии Львовского национального медицинского университета имени Данила Галицкого. scopic injection: Comparison to primary surgery. Investig Clin Urol. 2016 Jan;57(1):58-62. doi: 10.4111/ icu.2016.57.1.58.

15. Lee EK, Gatti JM, Demarco RT, Murphy JP. Longterm followup of dextranomer/hyaluronic acid injection for vesicoureteral reflux: late failure warrants continued followup. J Urol. 2009 Apr;181(4):1869-74; discussion 18745. doi: 10.1016/j.juro.2008.12.005. Epub 2009 Feb 23.

\section{Address for correspondence}

79010, Ukraine, Lviv,

Pecarskaya str., 69,

Lviv Danylo Halytsky National

Medical University,

Department of Pediatric Surgery,

Tel.: + 38-032-293-97-39

E-mail: nrostyslav@gmail.com

Rostyslav A. Nakonechnyy

\section{Information about the authors}

Nakonechnyy R.A. Post-graduate student of the pediatric surgery department, Lviv Danylo Halytsky National Medical University. 